\section{Diretriz para análises de impacto orçamentário de tecnologias em saúde no Brasil}

\author{
Guidelines for budget impact analysis of health \\ technologies in Brazil
}

\author{
1 Instituto de Avaliação de \\ Tecnologia em Saúde, Porto \\ Alegre, Brasil. \\ 2 Universidade Federal do Rio \\ Grande do Sul, Porto Alegre, \\ Brasil. \\ 3 Secretaria de Ciência \\ Tecnologia e Insumos \\ Estratégicos, Ministério da \\ Saúde, Brasília, Brasil. \\ ${ }^{4}$ Agência Nacional de \\ Vigilância Sanitária, Brasília, \\ Brasil. \\ Correspondência \\ A. L. Ferreira-Da-Silva \\ Instituto de Avaliação de \\ Tecnologia em Saúde. \\ Rua Ramiro Barcelos 2350, \\ sala 21507, Porto Alegre, \\ RS 90035-903, Brasil. \\ andre.ferreira@htanalyze.com
}

\begin{abstract}
Budget impact analysis (BIA) provides operational financial forecasts to implement new technologies in healthcare systems. There were no previous specific recommendations to conduct such analyses in Brazil. This paper reviews BIA methods for health technologies and proposes BIA guidelines for the public and private Brazilian healthcare system. The following recommendations were made: adopt the budget administrator's perspective; use a timeframe of 1 to 5 years; compare reference and alternative scenarios; consider the technology's rate of incorporation; estimate the target population by either an epidemiological approach or measured demand; consider restrictions on technologies' indication or factors that increase the demand for them; consider direct and averted costs; do not adjust for inflation or discounts; preferably, integrate information on a spreadsheet; calculate the incremental budget impact between scenarios; and summarize information in a budget impact report.
\end{abstract}

Health Planning Guidelines; Health Financing; Health Evaluation
Andre Luis Ferreira-Da-Silva 1,2 Rodrigo Antonini Ribeiro 1,2 Vânia Cristina Canuto Santos 3 Flávia Tavares Silva Elias 3 Alexandre Lemgruber Portugal d'Oliveira 4 Carisi Anne Polanczyk 1,2

Análise de impacto orçamentário: definições, importância e fundamentos

A análise de impacto orçamentário (AIO) pode ser definida como a avaliação das consequências financeiras advindas da adoção de uma nova tecnologia (intervenção) em saúde, dentro de um determinado cenário de saúde com recursos finitos 1,2. Diferentemente do que ocorre nas análises de custo-efetividade (ACE), em que geralmente se compara o efeito na relação custo e ganhos em saúde decorrentes da substituição de uma tecnologia por outra, na AIO é estimado o impacto econômico da incorporação (ou remoção) de uma nova intervenção, considerando-se o conjunto das tecnologias disponíveis para o problema de saúde em análise. Neste caso, estão compreendidos os custos da nova intervenção em si, custos de cointervenções, movimento de recursos associados às opções terapêuticas em uso e possíveis realocações de recursos para os casos em que a inclusão de uma nova tecnologia possa resultar em economias ao sistema de saúde 1,3,4,5,6.

O principal papel desse tipo de avaliação é a previsão do impacto financeiro da adoção de determinada tecnologia. Para tanto, integra os seguintes elementos: (1) o gasto atual com uma dada condição de saúde, (2) a fração de indivíduos elegível para a nova intervenção, (3) os custos diretos da nova intervenção e (4) o grau de in- 
serção da mesma após sua incorporação. Dessa forma, a AIO se constitui em uma ferramenta fundamental para os gestores do orçamento da saúde pública e suplementar, auxiliando a previsão orçamentária em um intervalo de tempo definido.

O Brasil vem apropriando-se dos instrumentos analíticos necessários às avaliações de novas tecnologias para a saúde 7 . Nesse contexto, tornou-se importante o desenvolvimento de recomendações nacionais para estudos de impacto orçamentário na área da saúde. No delineamento da presente proposta, foram consideradas as recomendações da International Society for Pharmacoeconomics and Outcomes Research (ISPOR) ${ }^{1} \mathrm{e}$ foram revisadas as diretrizes de outros países para a condução de estudos de impacto orçamentário 3,4,5,6. A metodologia para desenvolvimento de estudos de impacto orçamentário na área da saúde foi adaptada às necessidades brasileiras, ao longo de diversas sessões de apresentação e discussão entre os profissionais das instituições envolvidas. Adicionalmente, especialistas de todo o país foram consultados no processo de elaboração das presentes recomendações, e seus pareceres aprimoraram o texto final. Por fim, foram desenvolvidas planilhas eletrônicas interativas para auxílio no planejamento, na busca de dados e na condução dos estudos de impacto orçamentário desenvolvidos para o cenário brasileiro.

\section{O papel das análises de impacto orçamentário em comparação às análises de custo-efetividade}

A AIO deve ser vista como um complemento à ACE, e não como uma alternativa ou um estudo em substituição a esta 1,3. Enquanto a ACE estima, preferencialmente, em um horizonte temporal de tempo de vida, os custos e os benefícios de uma nova intervenção por indivíduo (unidades monetárias gastas para que um indivíduo tenha um ano de vida salvo), a AIO projeta os gastos que a incorporação da tecnologia em questão irá acarretar ao sistema em termos populacionais, considerando um horizonte de tempo geralmente mais curto (um a cinco anos).

Existe ainda uma similaridade metodológica entre os dois tipos de análise, as quais compartilham requerimentos de dados e alguns instrumentos analíticos. De fato, embora metodologicamente complexo, a análise de impacto orçamentário pode ser executada simultaneamente à ACE 8. Uma comparação mais detalhada entre as principais características desses dois tipos de análise está descrita na Tabela 1 .
Apesar de seus papéis complementares, é interessante observar que, eventualmente, os resultados de análises de AIO e ACE podem colocar tecnologias em posições diferentes na hierarquia de priorização para incorporação. Por exemplo, podemos simular um cenário no qual, para o tratamento de acidente vascular cerebral, existam duas novas tecnologias concorrentes e não sobreponíveis: medicamentos A e B. A ACE da opção A pode apresentar uma relação de custoefetividade incremental de R $\$ 15.000$ por ano de vida salvo, ao passo que a opção B, uma cifra de $\mathrm{R}$ \$ 25.000 para o mesmo benefício. Porém, como existem mais pacientes que preenchem os prérequisitos para a utilização do medicamento A do que para o medicamento B, o impacto orçamentário anual da incorporação de A foi estimado em R \$ 10.000.000 e, para a incorporação de B, em $\mathrm{R} \$$ 8.000.000. Nesse exemplo, apesar de o medicamento A ser mais eficiente, pois seu uso emprega de melhor maneira cada unidade monetária, o seu impacto financeiro é menos favorável. É escassa a literatura sobre como definir resoluções para esse tipo de dilema no processo de tomada de decisão pelo gestor. Nesses casos, no Brasil, analisam-se dimensões como magnitude do problema de saúde, potencial de inovação e transferência tecnológica, logística de incorporação nos serviços de saúde, potencial de adesão por pacientes e profissionais ${ }^{9}$. Internacionalmente, a rede de avaliação de tecnologias da União Europeia (EUnetHTA) criou um modelo de avaliação que inclui análise de domínios organizacionais, éticos, sociais e legais, úteis para resoluções do dilema apresentado 10 .

\section{Valoração das consequências diretas e indiretas}

Um ponto importante nas análises econômicas em saúde relaciona quais as consequências de determinada tecnologia devem ser valoradas para inclusão nos estudos. Para as análises de custo-efetividade, geralmente todos os custos diretos e indiretos são incluídos, a curto e longo prazo. Para as AIO, somente devem ser incluídas as consequências diretas sobre o sistema de saúde (sob a perspectiva do gestor) que resultam em mudanças práticas quando da incorporação ou retirada de uma tecnologia. Por exemplo, no cenário em que uma nova tecnologia reduz internações por eventos clínicos, estes devem ser considerados como economias reais diretas. Entretanto, isso não é consenso entre as diversas diretrizes internacionais e especialistas. 3,4,5,6.

Por outro lado, parece consensual que as consequências indiretas ou futuras em longo período não devam ser consideradas. Por exemplo, 
Comparação entre as características das análises de custo-efetividade e de impacto orçamentário.

\begin{tabular}{|c|c|c|}
\hline Características & Custo-efetividade & Impacto orçamentário \\
\hline Perspectiva recomendada & Sociedade/Terceiro pagador/Outras & Detentor do orçamento/Gestor \\
\hline Unidade dos resultados & $\begin{array}{l}\text { Custo por benefício, individual } \\
\text { (ex.: } \mathrm{R} \$ \text { por ano de vida salvo por } \\
\text { paciente tratado) }\end{array}$ & Custo absoluto, populacional \\
\hline Generalização dos resultados & Possível, com limitações & $\begin{array}{l}\text { Inadequada: estudos de impacto } \\
\text { orçamentário são elaborados para } \\
\text { circunstâncias específicas }\end{array}$ \\
\hline Comparação & $\begin{array}{c}\text { Tecnologias específicas: a nova } \\
\text { tecnologia será utilizada por toda a } \\
\text { coorte de intervenção }\end{array}$ & $\begin{array}{c}\text { Cenários, nos quais se pode projetar } \\
\text { o grau de incorporação da nova } \\
\text { tecnologia }\end{array}$ \\
\hline População em estudo & $\begin{array}{l}\text { Fechada: coorte de indivíduos } \\
\text { definidos a priori }\end{array}$ & $\begin{array}{l}\text { Aberta: indivíduos podem ser } \\
\text { incluídos ou excluídos ao longo } \\
\text { do tempo, considerando taxa } \\
\text { de incorporação da tecnologia, } \\
\text { incidência da doença, indicações } \\
\text { de tratamento e efeito do novo } \\
\text { tratamento na sobrevida }\end{array}$ \\
\hline Horizonte temporal & $\begin{array}{l}\text { Preferencialmente todo o tempo de } \\
\text { vida }\end{array}$ & $\begin{array}{l}\text { Conforme conveniência do gestor } \\
\text { (geralmente } 1-5 \text { anos) }\end{array}$ \\
\hline Utilização de taxa de desconto & Extremamente recomendada & Não recomendada \\
\hline
\end{tabular}

uma intervenção que reduza a mortalidade por uma determinada enfermidade poderá provocar outros custos relacionados a doenças futuras para esta população, em virtude da maior sobrevida e do envelhecimento. Essas consequências indiretas não devem ser consideradas nas estimativas de impacto orçamentário.

\section{Delineamento dos estudos de impacto orçamentário}

O desenho geral dos estudos de impacto orçamentário é ilustrado na Figura 1, adaptado das diretrizes da ISPOR ${ }^{1}$. Nela está representada uma visão geral de uma AIO, na qual dois (ou mais) cenários de uma doença são comparados em relação aos seus custos. O cenário de referência consiste em uma representação da realidade atual da doença em questão, incluindo estimativas do tamanho da população de interesse (a partir de incidência/prevalência/subgrupos) e os recursos utilizados para o seu manejo. No cenário alternativo, devem ser considerados os efeitos da nova intervenção, que pode ser preventiva, diagnóstica ou terapêutica, no custo do manejo da doença. O impacto orçamentário é, basicamente, a diferença entre os custos dos dois cenários. As etapas na execução de uma AIO serão descritas em maior detalhe nas próximas seções do texto.

\section{Métodos para estimar o impacto orçamentário}

De forma simplificada, o custo do tratamento de uma determinada doença equivale a multiplicar o número de indivíduos doentes com indicação de tratamento pelo custo dos tratamentos que estão sendo avaliados. O impacto orçamentário incremental consiste na diferença de custos entre o cenário do novo tratamento e o cenário de referência (que reflete as práticas terapêuticas atuais, sem a nova intervenção):

\section{impacto orçamentário incremental = (NiNt x CtNt) - (NtA x CttA)}

sendo:

$\mathrm{NiNt}=$ número de indivíduos usando o novo conjunto de tratamentos formado a partir da incorporação da nova intervenção em estudo;

$\mathrm{CtNt}=$ custo total do novo tratamento;

$\mathrm{NtA}=$ número de indivíduos usando o atual conjunto de tratamentos disponível;

$\mathrm{CttA}=$ custo total do tratamento atual.

Existem duas principais estratégias de integrar as informações sobre estimativa da popu- 


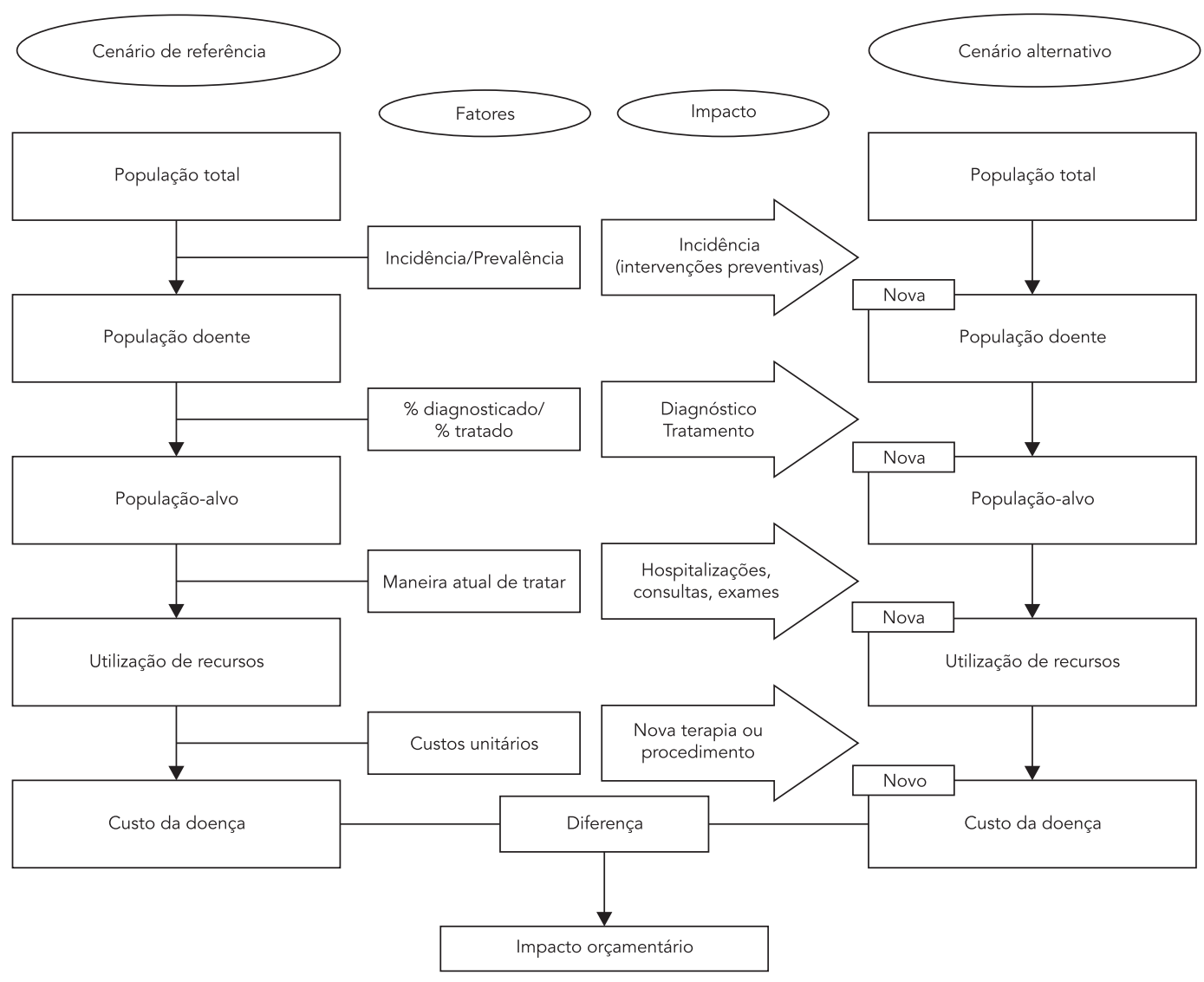

lação de interesse com as estimativas de custos de forma comparativa entre diferentes cenários 1,3,4,5,6,11: (1) modelos estáticos (planilha eletrônica determinística e modelo de árvore de decisão simples); (2) modelos dinâmicos (Modelo de Markov e outros modelos dinâmicos, como planilha eletrônica dinâmica, simulação de eventos discretos, outros).

\section{Modelos estáticos}

A modelagem estática consiste na multiplicação simples do custo individual da nova intervenção pelo número de indivíduos com indicação de uso (para as doenças crônicas) ou de episódios da doença com indicação de tratamento (para as doenças agudas). Trata-se do método de mais fácil execução e também o mais amplamente difundido e utilizado, o qual pode ser executado em planilhas eletrônicas ou em árvores de decisão simples. É também chamado de método determinístico, pois são aplicadas estimativas "determinadas” dos parâmetros necessários à análise, como parâmetros epidemiológicos e custos 1 . A despeito de sua popularidade, a modelagem estática apresenta algumas limitações, como a restrita capacidade de simular movimentos complexos de mercado ao longo do tempo, ou de modelar aspectos dinâmicos de doenças, como no caso de doenças agudas ou de doenças crônicas com frequentes exacerbações e remissões.

\section{Modelos dinâmicos}

A modelagem dinâmica, por sua vez, consiste na elaboração de um modelo de estados transicionais (modelo de Markov) ou de outro método, para a simulação de coortes. Esse modelo é capaz 
de simular a dinâmica da doença incorporando as diferentes probabilidades de transição entre os estados de saúde e também de simular as possíveis transições entre as opções terapêuticas disponíveis ao longo do tempo. Aqui, daremos destaque ao uso dos modelos de Markov para a modelagem dinâmica de estados de saúde e de transições entre as opções terapêuticas 8 .

\section{a) Modelos de Markov}

Dentre os métodos dinâmicos, os modelos mais utilizados são os de Markov. Esse tipo de modelagem, também chamada de modelo de estados transicionais, é amplamente utilizado na literatura de ACE. Uma descrição mais detalhada do emprego dessa metodologia em ACE foge do escopo deste documento, sendo sugerida a leitura do artigo de Sonnenberg \& Beck 12 para maior aprofundamento. Um detalhe característico desse tipo de modelo nas ACE é o uso dos estados de Markov no cálculo dos custos e da efetividade para populações fechadas e de tamanhos indeterminados, isto é, aquelas em que o número de indivíduos na população não se altera ao longo do tempo (exceto pela incidência de óbitos) e o tamanho da população não é quantificado.

Por outro lado, nas análises de impacto orçamentário, os objetivos são diferentes: é desejável trabalhar com populações abertas, aquelas em que, ao longo do tempo, pode haver saída de indivíduos (não por óbito, mas por outros motivos, como migração, mudança para um estado de doença não mais elegível para o tratamento), ou entrada (por natalidade ou incidência de doença). Em adição, é necessário que o tamanho da população seja delimitado, correspondendo à prevalência de pessoas com dada condição na população-alvo do gestor do orçamento.

A modelagem das coortes abertas, necessárias para casos selecionados de análises de impacto orçamentário, pode ser executada mediante softwares de análise de decisão como Treeage (TreeAge Software, Inc., http://www.treeage.com), ou pode ser construída em planilhas eletrônicos como Excel (Microsoft Corp., Estados Unidos).

Além dos modelos de Markov, há ainda as análises realizadas por simulação de eventos discretos. São outra possibilidade de modelagem que contemplam entrada e saída de indivíduos no modelo e contam com algumas particularidades que permitem maior detalhamento do que os modelos de Markov. Características como análise centrada em indivíduos, e não em estados de saúde, e de tratamento do tempo como discreto, e não intervalar, fazem com que tais análises tenham maior versatilidade 1 . Contudo, requerem uma maior quantidade de dados para sua execu- ção e exigem uma complexidade computacional muito maior.

\section{Escolha do método apropriado à análise de impacto orçamentário}

A escolha do método apropriado para modelagem do impacto orçamentário dependerá do grau de precisão com que se busca reproduzir as condições de mercado, além da complexidade da doença e da intervenção em análise 1,8. De um modo geral, a maioria das AIO pode ser executada em planilhas eletrônicas, desde que os pressupostos assumidos para caracterização da população-alvo e da composição dos custos estejam nelas representados. O presente documento é voltado principalmente à AIO por meio de planilhas eletrônicas. Seja qual for o método escolhido, a opção por uma determinada técnica de modelagem deve ser declarada e justificada.

O modelo de impacto orçamentário pode ser desenvolvido independentemente da ACE, estratégia que recomendamos. É possível a inclusão de uma modelagem para impacto orçamentário em uma árvore de decisão ou modelo de Markov, desenvolvido para ACE, entretanto raramente os estudos de impacto orçamentário demandam uma abordagem tão complexa.

\section{Recomendações para o desenvolvimento de uma análise de impacto orçamentário no cenário brasileiro}

\section{Critérios e definições fundamentais \\ da análise}

O primeiro passo na elaboração de uma AIO consiste na indicação das características básicas da análise: delimitar a doença em questão, o novo tratamento em avaliação, a perspectiva da análise, o horizonte temporal em anos e os nomes para até quatro cenários em comparação. O objetivo dos diferentes cenários é simular variados graus de inserção do novo medicamento.

\section{Perspectiva da análise}

A perspectiva da análise, recomendada pelas diretrizes internacionais e também indicada para o Brasil, é a do gestor do orçamento de um sistema de saúde, regional ou nacional. No caso brasileiro, as perspectivas mais comumente adotadas serão as do Sistema Único de Saúde (SUS), em suas diferentes esferas, e do sistema de saúde suplementar (planos e convênios de saúde privados). O gestor responsável pela encomenda ou desenvolvimento da AIO em questão deve 
ser devidamente nomeado: Secretarias Estaduais ou Municipais da Saúde, Ministério da Saúde ou programas específicos com orçamentos próprios. No contexto de uma AIO desenvolvida sob a perspectiva do sistema de saúde suplementar, o nome do provedor de saúde deverá ser informado. Uma AIO também pode ser desenvolvida conforme a perspectiva de gestores locais do sistema de saúde, como gestores de hospitais e de centros de saúde.

\section{- Horizonte temporal}

Recomenda-se o período de um a cinco anos para o horizonte temporal da análise, com as estimativas de impacto orçamentário relatadas ano a ano. A preferência por um horizonte temporal de um ou de cinco anos deve considerar a expectativa de difusão da tecnologia no sistema de saúde a partir de sua implementação, optandose pelo período mais longo para as tecnologias cuja estabilização de demanda no mercado exija mais tempo.

\section{Cenários a serem comparados}

As AIO baseiam-se na comparação de dois ou mais cenários, os quais são representações de diferentes condições de mercado. Cada cenário é composto por diferentes proporções de uso para as diferentes opções terapêuticas existentes em um sistema de saúde.

Denomina-se "cenário de referência" o conjunto de opções terapêuticas atualmente disponíveis para o tratamento da doença de interesse no sistema de saúde para o qual se aplica a AIO. Esse é, portanto, o cenário que representa o atual padrão de uso dos tratamentos disponíveis para uma dada enfermidade em um determinado sistema de saúde.

Na definição do cenário de referência, devem-se listar as opções terapêuticas já usadas ao lado do percentual do mercado presentemente ocupado por cada uma delas. A proporção do uso de um determinado medicamento em um dado mercado pode ser uma informação particularmente difícil de obter. A Tabela 2 apresenta as diferentes alternativas de fontes confiáveis para essa informação.

Embora as diferentes fontes de informações sobre as atuais condições do mercado sejam aceitáveis para a definição do cenário de referência e dos cenários alternativos, obviamente prefere-se informação procedente de órgãos oficiais ou de publicações científicas. É natural que a realização de um estudo de prevalência do uso das diferentes opções terapêuticas forneça as estimativas mais precisas, entretanto os custos associados à realização de um subestudo especificamente para esse fim e o tempo demandado na sua execução raramente são compensados pela maior qualidade da informação obtida. Por fim, considera-se que o uso do painel de especialistas na caracterização do mercado atual deva ser adotado quando outras fontes de dados não estiverem disponíveis. O painel de especialistas revela-se útil na estimação das condições de mercado futuras.

Ao utilizar as informações sobre as diferentes terapias que compõem os cenários a serem comparados, as intervenções envolvidas devem ser identificadas, bem como a proporção esperada do uso de cada fármaco (ou outra tecnologia) nos cenários de referência e alternativos. Adicionalmente à caracterização da nova intervenção que está sendo avaliada, deve-se elaborar uma revisão e descrição das principais modalidades terapêuticas atualmente empregadas no sistema de saúde. Os tratamentos atualmente empregados devem ser descritos considerando-se os mesmos itens usados para a nova intervenção.

A caracterização do atual cenário para o manejo da doença de interesse deve ser embasada, preferencialmente, em diretrizes e recomendações aplicadas localmente ou em estudos publicados sobre os padrões de prescrição para a população-alvo no território de interesse. A definição das quotas do mercado atual pode ser difícil quando esse tipo de estudo não estiver disponível. A adoção das estimativas de quotas de mercado (atual e projeções) encontradas em outros países é uma alternativa para a obtenção dessa informação. Um painel de especialistas (por meio do método Delphi, por exemplo) pode ser empregado para validar e adaptar estimativas importadas ao mercado nacional.

\section{- Difusão e velocidade de incorporação da nova tecnologia}

Apesar de esperado que novas tecnologias e terapias disponíveis sejam rapidamente incorporadas à prática clínica, é reconhecido que existe demora até sua aplicação plena. A disponibilidade de equipamento, o treinamento e a capacitação de pessoal, a operação de todo sistema, além de hábitos dos médicos e pacientes, podem alterar a taxa de difusão de novas tecnologias. Segundo estudos internacionais nesta área, algumas terapias são rapidamente incorporadas - pelas facilidades, benefícios, pressão de mercado etc. -, enquanto outras têm sua incorporação retardada ou, até mesmo, não plenamente considerada. Esses parâmetros precisam ser incluídos na estimativa das unidades anuais de consumo da terapia. 
Possíveis fontes para estimar as quotas de mercado ocupadas pelas diferentes intervenções existentes para o tratamento de uma mesma enfermidade.

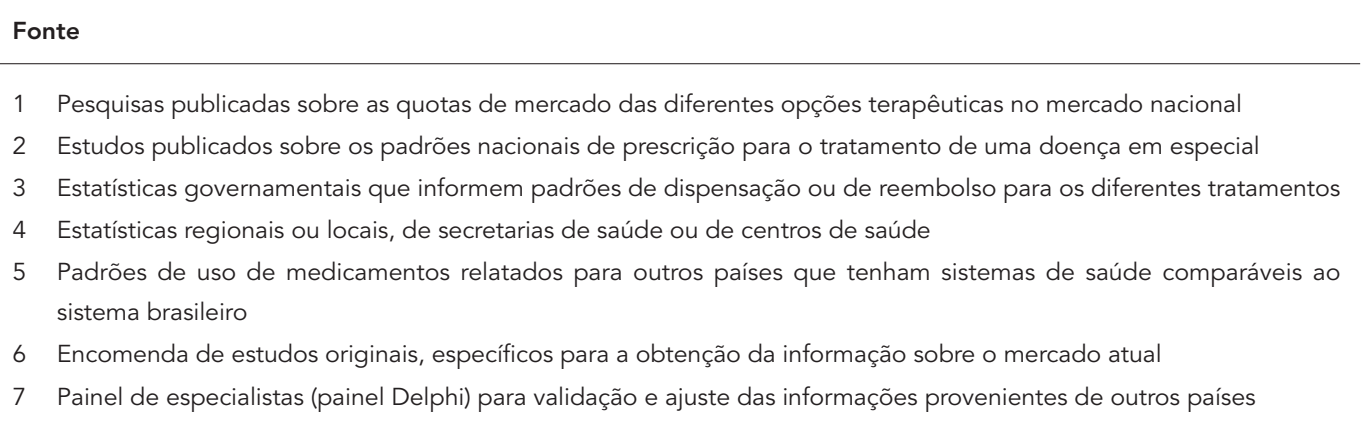

\section{Delimitação da população de interesse}

Nas AIO, existem basicamente dois métodos para a delimitação da população de interesse: o método epidemiológico e o método da demanda aferida (Figura 2).

No método epidemiológico, parte-se dos dados epidemiológicos oficiais mais atualizados e precisos disponíveis. Primeiramente, define-se o território de interesse, a população que nele habita e o percentual dessa população que corresponde à faixa etária de interesse.

A unidade de análise é definida de acordo com a natureza da enfermidade:

- Doenças agudas e exarcebações agudas crônicas: a unidade de análise é o episódio, e a estimativa epidemiológica deverá ser baseada na incidência de episódios;

- Doenças crônicas: a unidade de análise é o indivíduo e a estimativa epidemiológica deverá ser baseada na prevalência de casos da doença.

Como definição geral, para fins da AIO, podem-se considerar doenças agudas aquelas com duração inferior a 12 meses e doenças crônicas aquelas com duração de 12 ou mais meses. Essas definições não são absolutas, e a escolha de caso ou episódio como unidade de análise dependerá da natureza da doença em questão e do tratamento avaliado.

Em seguida, aplica-se a melhor estimativa da prevalência (ou da incidência) da doença para aquele território, a fim de se obter, dentro da faixa etária de interesse, o número esperado de indivíduos (ou de episódios) portadores do problema de saúde em estudo. A consideração simultânea da prevalência e da incidência da doença pode ser apropriada nos casos em que ambas forem significativas e afetarem sensivelmente a evolução do tamanho da população-alvo no horizonte temporal definido. Incertezas quanto às estimativas desta população podem ser avaliadas por intermédio de uma análise de sensibilidade, cujos métodos serão descritos adiante.

O método epidemiológico tem a vantagem de estimar de forma mais abrangente todos os indivíduos cobertos por um determinado sistema de saúde que poderiam se beneficiar do tratamento em avaliação. Por basear-se em dados estatísticos e em estimativas de parâmetros epidemiológicos, o número calculado de pacientes tende a ser maior do que o contabilizado quando há uma estatística oficial, em razão de subnotificações ou de pacientes que simplesmente não estão cientes de seu diagnóstico. Além disso, nem todos os pacientes portadores de uma determinada doença fazem seu tratamento e acompanhamento pelo sistema público de saúde, de modo que a proporção que realiza tratamento por esse sistema deverá ser considerada quando as análises forem efetuadas sob determinada perspectiva. Deve-se proceder de forma análoga quando a AIO for desenvolvida sob a perspectiva do sistema de saúde suplementar.

Por outro lado, algumas vezes, o gestor de um sistema de saúde dispõe de alguma estimativa da população de interesse, não sendo conveniente ou necessário que sejam feitas novas previsões pelo método epidemiológico. Quando isso ocorre, a população de interesse é definida pelo método da demanda aferida. Na literatura internacional, essa abordagem é chamada de claims data-based model (diretrizes canadenses) ${ }^{3}$ ou de market share approach (diretrizes australianas) 5 e pode ser feita de duas formas: (1) uso da contagem de pacientes cadastrados, quando existe algum tipo de sistema de cadastro específico; (2) uso do número histórico, ou de um determinado ano, de pedidos de reembolso por um tratamento 


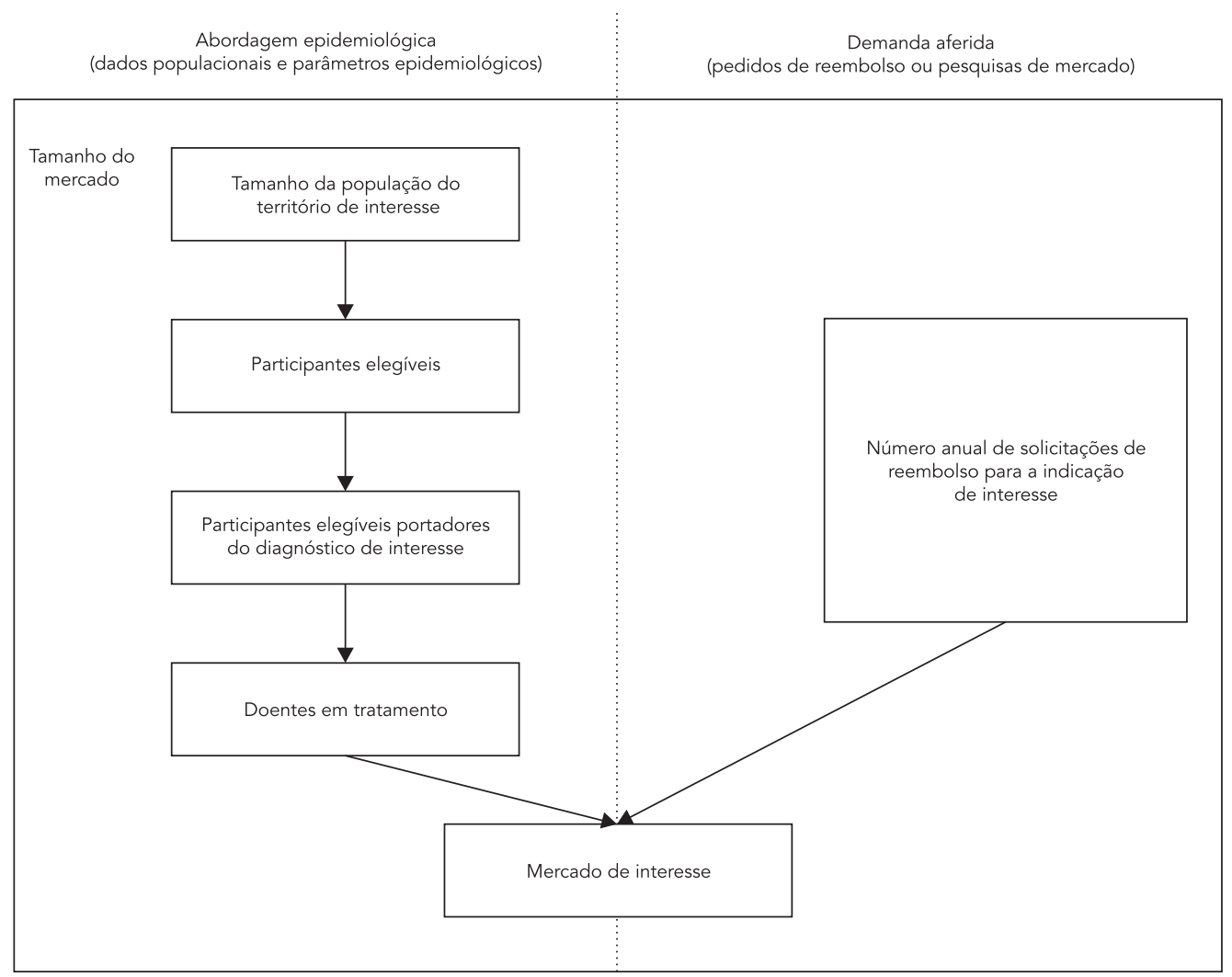

médico em um plano de saúde específico, como o possível futuro número de reembolsos pela intervenção para a qual está sendo conduzida a AIO.

\section{Restrições e demandas adicionais}

Na condução de uma AIO, deve-se estabelecer qual será, exatamente, a indicação do novo tratamento no sistema de saúde. A seguir, com base em estudos clínicos e epidemiológicos, deve-se estimar a proporção dos pacientes que se enquadram na indicação estabelecida.

Nem todos os indivíduos identificados pelo método epidemiológico ou da demanda aferida como portadores do problema de saúde em questão têm indicação de uso da nova tecnologia em análise. Frequentemente, por razões tanto de ordem técnica, quanto de limitações orçamentárias, os gestores de sistemas de saúde necessitam fazer uso de restrições de acesso à nova intervenção como forma de assegurar que esta seja utilizada pelos indivíduos que, de fato, terão o maior benefício terapêutico, conforme os estudo clínicos que embasam a indicação do novo tratamento. Dessa forma, impede-se, ao mesmo tempo, que a nova intervenção seja desviada para pacientes que não têm indicação de tratamento conforme a melhor evidência científica disponível.

Nas situações em que a implementação em larga escala de uma nova tecnologia da saúde não seja viável por razões orçamentárias, ACE poderão indicar que tipo de paciente obtém a razão de custo-efetividade mais favorável. Tal critério pode ser usado para restringir o acesso àqueles que usufruem do maior benefício comparativamente aos custos.

\section{Dinâmica da doença}

A natureza da doença-alvo para o novo tratamento tem grande influência nas projeções de im- 
pacto orçamentário. Doenças crônicas com incidência não desprezível e mortalidade que não supere sua incidência tendem a se acumular na população. Inserem-se nesse quadro, por exemplo, as doenças cardiovasculares, osteomusculares, além das condições consideradas fatores de risco, como hipertensão arterial e diabetes mellitus. Nesse caso, a prevalência de ocorrência da doença corresponde à informação principal para se estimar a população de interesse. No entanto, a incidência de novos casos (ou de episódios) e a mortalidade também devem ser consideradas, o que é particularmente importante nas situações em que o tratamento em avaliação é capaz de reduzir a mortalidade da doença, aumentando sua prevalência e, consequentemente, o impacto orçamentário.

A consideração da mortalidade na análise de impacto orçamentário realizada em planilha eletrônica não pode ser feita em todas as circunstâncias e não é considerada obrigatória. Para os casos em que a mortalidade associada à doença de interesse e o efeito do tratamento sobre esta forem significativos, pode ser necessária a construção de um modelo dinâmico, como um modelo de Markov.

Para doenças crônicas que apresentem baixa incidência e mortalidade associadas, o dado de prevalência pode ser suficiente para a modelagem de impacto orçamentário. Esse é o caso, por exemplo, da artrite reumatóide.

No que se refere às doenças agudas, a modelagem é mais bem conduzida quando se considera o episódio, e não o indivíduo, como unidade de análise. Nesses casos, a incidência de novos episódios e a mortalidade são os parâmetros de maior influência nas estimativas de impacto orçamentário.

\section{Custos do tratamento}

Nas AIO, todos os custos considerados devem refletir a perspectiva do gestor do orçamento da saúde a quem o estudo se destina, isto é, o valor que, de fato, será desembolsado pelo gestor por ocasião da adoção da nova tecnologia da saúde. Essa perspectiva opõe-se às ACE, que adotam o conceito de custo de oportunidade, que se refere ao benefício potencialmente perdido ao adotarse uma intervenção em lugar de outra.

\section{- Custos que devem ser considerados: custo direto do pacote terapêutico}

Recomenda-se que, em uma intervenção, somente os custos diretos sejam considerados, o que inclui os custos da nova tecnologia em si e aqueles diretamente associados ao seu uso, como medicamentos adjuvantes ou tratamento de eventos adversos. Assim, o custo de cada intervenção incluída na análise refletirá o custo de todo o "pacote" terapêutico associado àquela intervenção. Esse custo combinado deve ser calculado não somente para a nova tecnologia da saúde que motiva a AIO, mas também para as outras intervenções que compõem o cenário de referência, além dos diferentes cenários alternativos que serão modelados.

Desse modo, por exemplo, ao estimar-se o custo de um medicamento quimioterápico, devem ser considerados os custos associados (internações para aplicação, medicamentos adjuvantes, como corticosteroides, e medicamentos usados para prevenir ou tratar eventos adversos). Por outro lado, custos não diretamente relacionados às intervenções avaliadas não devem ser considerados na AIO. Igualmente, aqueles decorrentes de consequências indiretas ou tardias do tratamento também não devem ser incluídos.

Na determinação de quais custos associados são significativos e devem, por isso, ser incluídos na análise, é importante um bom entendimento dos aspectos práticos da administração da intervenção e dos eventos adversos associados, que podem produzir custos em seu manejo. Essas informações podem ser obtidas por meio da consultoria com especialistas e da revisão dos estudos clínicos, nos quais foram documentadas as frequências dos eventos adversos menores e maiores da intervenção. A Tabela 3 apresenta os principais custos a serem considerados para cada uma das intervenções incluídas na análise.

Outra possível fonte para estimativa dos custos seria a condução de uma pesquisa de custos original, obtendo-se dados primários, por amostragem, envolvendo entrevistas com os profissionais da área da saúde diretamente responsáveis pela administração da intervenção em estudo. Embora essa estratégia permita uma definição muito mais precisa dos custos envolvidos, os recursos financeiros e o tempo demandado para sua execução acabam por torná-la pouco prática.

\section{- Estimativa do consumo das intervenções analisadas por período de tempo}

Ao se estimar a quantidade média da nova intervenção requerida por paciente, por período de tempo (mês ou ano, geralmente), algumas fontes de informação podem ser adotadas: estimativa com base nos estudos clínicos da intervenção analisada; no consumo atual de intervenção semelhante, para a qual se cogita a substituição pela nova intervenção em estudo; no consumo da intervenção por paciente em países onde a 
Custos diretamente relacionados à tecnologia da saúde analisada e indicação de fontes para suas estimativas.

\begin{tabular}{|c|c|}
\hline Custos & Possíveis fontes de dados \\
\hline \multicolumn{2}{|l|}{ Custos da intervenção em si } \\
\hline Medicamentos: valor unitário e número de unidades & Preços de fábrica \\
\hline por mês ou ano & Preços de aquisição pagos pelo gestor (incluir tributação \\
\hline Dispositivos: custo do dispositivo, custo com implante, & quando aplicável) \\
\hline custo de manutenção no horizonte temporal de & Preço médio de mercado \\
\hline interesse & Banco de preços do Ministério da Saúde \\
\hline \multirow{2}{*}{\multicolumn{2}{|c|}{$\begin{array}{l}\text { Procedimentos: custo do procedimento (material e } \\
\text { recursos humanos) }\end{array}$}} \\
\hline & \\
\hline \multicolumn{2}{|l|}{ Teste diagnóstico: custo de equipamentos, custo } \\
\hline \multicolumn{2}{|l|}{ mensal de insumos } \\
\hline \multicolumn{2}{|l|}{ Custos diretamente associados à intervenção } \\
\hline Tratamentos adjuvantes: custo unitário e unidades por & Tabela de repasses do SUS \\
\hline mês ou ano & Tabelas de reembolso dos planos de saúde \\
\hline Eventos adversos leves: frequência e custo do manejo & Preços praticados em outros países, com conversão \\
\hline Eventos adversos graves: frequência e custo do manejo & cambial e ajuste para o poder de compra \\
\hline Custo decorrente de consultas médicas & Estudos de custo realizados em conjunto com ensaios \\
\hline Custo de exames laboratoriais & clínicos \\
\hline Custo de hospitalizações & \\
\hline
\end{tabular}

intervenção já foi adotada; na dosagem média necessária por paciente, por mês, de acordo com os dados fornecidos pelo fabricante; e na opinião de especialistas.

No caso de AIO de medicamentos, os dados sobre a frequência mensal de dispensação de drogas, o reembolso por paciente ou os pedidos de reembolso por medicamentos prescritos para uma população definida podem ser usados nas estimativas da necessidade média mensal do novo medicamento.

\section{- Aplicação do método da equivalência terapêutica na comparação de custos}

As diferentes intervenções avaliadas em uma AIO podem ter esquemas posológicos diferentes ou custos únicos relacionados à sua implementação (como nos casos de dispositivos e procedimentos). Por essa razão, faz-se necessária a aplicação do método da equivalência terapêutica, que torna comparável o custo anual das diferentes intervenções. Esse método consiste na estimativa dos custos totais associados às intervenções em um período de tempo determinado (por exemplo, mensal ou anual).

Assim, intervenções como o implante de dispositivos ou a realização de procedimentos médicos podem ser contabilizados pelo valor anual demandado. Na composição desse custo anual, custos periódicos de manutenção podem ser incluídos também, com base anual ou relatados por períodos de tempo menores, quando apropriado.

\section{- Consideração de custo com medicamentos genéricos e efeito da nova intervenção no uso de outras medicações}

Nos casos das AIO de fármacos desenvolvidas sob a perspectiva do sistema de saúde suplementar, quando se antecipa que o medicamento avaliado estará disponível sob a forma de genérico no horizonte temporal da análise, o custo estimado desse medicamento deverá ser incluído no modelo. Naturalmente, em todas as circunstâncias, as incertezas nas estimativas de custos poderão ser consideradas em uma análise de sensibilidade.

Caso se antecipe que a nova intervenção substituirá uma outra em uso, isso deve ser representado em um dos cenários alternativos, e os custos que deixarão de existir com a substituição deverão ser considerados. De forma análoga, intervenções que tendem a ter seu uso aumentado ao se adotar uma nova tecnologia da saúde (como, por exemplo, um fármaco adjuvante fundamental) também devem ter seu custo contabilizado nas estimativas de impacto orçamentário. 


\section{- Cálculo do custo por cenário}

Nessa etapa, são calculados os custos médios anuais por cenário, por paciente, por ano, considerando a composição de cada cenário e a taxa de incorporação da nova tecnologia. Esse custo é obtido multiplicando-se o custo total anual da intervenção pela proporção dos pacientes que usará cada uma das intervenções em cada cenário.

\section{Custos evitados}

A incorporação de uma nova tecnologia assume que existe um benefício clínico para pacientes e populações. Esse benefício pode ser mensurado em termos econômicos, podendo, também, ser considerado na AIO.

O objetivo dessa etapa é estimar custos que podem ser evitados em decorrência do emprego da nova tecnologia que está sendo avaliada na AIO. Um exemplo disso seriam custos evitados pela redução da necessidade de hospitalizações para intervenções que demonstrem a capacidade de redução da ocorrência desse desfecho. Outros exemplos incluem a redução da necessidade de cirurgias, de hemodiálise ou do uso de determinados medicamentos de alto custo.

As informações necessárias à contabilização dos custos evitados devem ser extraídas do principal estudo clínico que tenha demonstrado eficácia na redução do desfecho cujo custo será evitado. Excetuam-se os dados de custos, os quais devem ser estimados com base em fontes pertinentes à perspectiva da análise.

A estimativa de custos relacionados às doenças pode demandar tempo e esforço considerável, nem sempre possível em todos os momentos. Esses custos podem ser estimados valendo-se de diferentes metodologias, dependendo das fontes de dados disponíveis e do seu detalhamento. Os custos hospitalares e de serviços de saúde podem ser bastante precisos valorando-se custo unitário e diário de cada paciente com determinada condição clínica (micro-costing ou bottom-up), ou ser obtidos mediante macroestimativas (average per diem cost ou top-down), na quais dados individuais não são considerados, e o custo total é dividido pelo número de atendimentos.

Para algumas doenças e cenários clínicos, pode ser muito relevante a obtenção de dados detalhados, levando-se em consideração gravidade e estadiamento de condições de saúde. Foge ao escopo desta diretriz descrever tais técnicas, vantagens e desvantagens de uso. Maior detalhamento pode ser encontrado nas diretrizes metodológicas para estudos de avaliação econômica de tec- nologias para o Ministério da Saúde, além de em livros e textos sobre economia da saúde 13,14.

\section{Ajustes econômicos}

Nas AIO, os ajustes para a inflação e para taxa de descontos não são rotineiramente recomendados. As justificativas para a não realização desses ajustes incluem (1) o horizonte temporal curto das AIO e (2) o fato de que o valor obtido em uma AIO corresponde a um valor presente usado nas estimativas orçamentárias do gestor, considerando que o orçamento é um valor financeiro gasto no presente e que não é reajustado pela inflação, nem influenciado por descontos.

O desconto é o ajuste econômico que busca transformar gastos futuros em valores presentes, considerando a tendência de desvalorização do dinheiro, além daquela decorrente do efeito da inflação. A teoria que embasa esse ajuste é a da preferência temporal, segundo a qual os indivíduos dão mais valor ao dinheiro no presente do que no futuro, mesmo após ajuste inflacionário. Levando-se em conta que as projeções de impacto orçamentário representam fluxos financeiros ao longo do tempo e que o objetivo da AIO é fornecer informações para o planejamento financeiro presente, recomendamos que a taxa de descontos não seja rotineiramente considerada, exceto em situações especiais, como uma AIO projetada para um horizonte temporal incomumente longo.

Por outro lado, as diretrizes internacionais não recomendam ajuste para a inflação, sob a alegação de que, tipicamente, os preços dos medicamentos não tendem a subir 3,5. Essa afirmação pode não ser verdadeira na realidade brasileira, e o ajuste para a inflação (ou deflação) pode ser realizado de forma opcional, quando se projeta significativa incidência de inflação ou deflação nos preços dos componentes de custos das intervenções analisadas no horizonte temporal de interesse.

\section{Integração das informações e comparação entre cenários}

A próxima etapa corresponde à integração de todas as informações inseridas no modelo anteriormente para que se obtenha uma projeção orçamentária ano a ano no horizonte temporal de interesse. O método utilizado consiste na multiplicação do custo médio anual por paciente, para cada cenário, pela estimativa da população de interesse. Nos anos subsequentes, o tamanho da população é recalculado nos casos em que a 
incidência da doença e sua letalidade são consideradas. Caso o modelo tenha sido ajustado para inflação ou descontos, os valores financeiros ajustados também deverão ser apresentados.

Cenários alternativos - impacto orçamentário de cada cenário

Adicionalmente ao cenário de referência, que reflete os atuais custos do gestor com o tratamento de uma dada enfermidade, recomendase que um a três cenários alternativos sejam simulados. Cada cenário alternativo deverá ser concebido com base nas projeções das quotas de mercado e da taxa de incorporação da tecnologia avaliada.

A comparação dos custos dos cenários alternativos com os custos do cenário de referência informa o impacto orçamentário. Desse modo, é obtida uma projeção orçamentária ano a ano no horizonte temporal de interesse para cada um dos cenários modelados.

Recomenda-se que a integração das informações relacionadas ao tamanho da população, às quotas de mercado na composição dos cenários e aos custos das opções terapêuticas seja realizada em uma planilha eletrônica interativa. Nos casos em que a AIO for considerada demasiadamente complexa para uma modelagem em planilha eletrônica, modelos em árvores de decisão simples, modelos de Markov e modelos em simulação de eventos discretos são alternativas aceitáveis.

\section{Impacto orçamentário incremental}

O impacto orçamentário incremental ou diferencial consiste no resultado principal de uma AIO e sempre deverá ser calculado e apresentado. É esse número que informa ao gestor o custo adicional decorrente da incorporação de uma nova tecnologia, em comparação ao cenário de referência, que representa os custos que já vêm sendo incluídos.

O impacto orçamentário diferencial consiste na simples diferença de valores de impacto orçamentário entre dois cenários. Recomenda-se calcular o impacto orçamentário incremental de todos os cenários alternativos em relação ao cenário de referência. É possível que o valor resultante seja negativo, situação na qual a adoção de um dos cenários significaria economia monetária.

\section{Avaliação das incertezas do modelo}

Recomenda-se que as incertezas na estimação dos parâmetros do modelo sejam avaliadas nu- ma análise de sensibilidade; esta deve ser realizada de forma sistemática e abrangente. Os métodos usados na avaliação de incertezas nas análises econômicas comparativas, do tipo ACE, nem sempre podem ser usados nas AIO.

Quanto às análises de sensibilidade, existem diversas técnicas para a sua execução: análise de sensibilidade univariável, multivariável, probabilística e por cenários. Todas podem ser efetuadas e são aceitáveis, entretanto, pela praticidade na execução e pela fácil interpretação dos resultados, recomendamos a análise de sensibilidade por cenários.

\section{Análise de sensibilidade por cenários}

Consiste em recalcular toda a AIO após alteração em uma ou mais variáveis do modelo, dentro de uma gama de valores plausíveis e de interesse ao gestor do sistema de saúde. Na prática, trata-se de modificar as variáveis da planilha, da árvore de decisão ou do modelo dinâmico cujas estimativas de valor sejam consideradas de menor precisão.

Recebe esse nome porque cada recálculo da AIO consiste em um novo cenário, podendo haver variações na composição dos cenários na curva de difusão da tecnologia, no tamanho da população-alvo, nos fatores que influenciam a utilização da nova tecnologia, nos custos e na presença ou não de ajustes econômicos.

Os principais componentes da AIO a serem testados na análise de sensibilidade incluem os seguintes: estimativas do custo da nova tecnologia da saúde; estimativa do tamanho da população de interesse; estimativa das quotas de mercado e de sua distribuição futura; estimativa da curva de difusão da nova tecnologia; variações nos preços de medicamentos quando se antecipa inclusão de medicamento genérico no mercado; variação no preço de medicamentos, quando se antecipa o ingresso no mercado de outro fármaco que compete pelo mesmo mercado; variação no tamanho da população de interesse por uso off-label da intervenção estudada (falha na restrição) ou outras demandas, como por decisão judicial.

Os novos cenários concebidos para a análise de sensibilidade devem ser brevemente descritos, assim como a variação observada nas estimativas do impacto orçamentário.

Quanto à definição da gama de variação dos valores usados na análise de sensibilidade por cenários, os seguintes métodos podem ser utilizados: modificação da variável em 25\% (ou outro percentual arbitrário) para mais e para menos; modificação da variável dentro de um intervalo de confiança relatado no estudo em que foi esti- 
mada; melhor cenário versus pior cenário (para uma ou mais variáveis em particular); estimativa da gama de valores plausíveis para a variável através de um painel de especialistas.

Embora dados da literatura ou dos registros oficiais do mercado de interesse sejam as fontes de informações preferenciais, os estudos de impacto orçamentário e suas análises de sensibilidade por cenários requerem, frequentemente, o uso do painel de especialistas (painel Delphi) para estimativa de valores médios e intervalo de dispersão de algumas variáveis. Isso ocorre porque algumas informações necessárias à condução das AIO são, na verdade, estimativas futuras da evolução da população-alvo, do comportamento do mercado e dos valores financeiros da tecnologia em avaliação e de suas opções concorrentes. Como não existe forma precisa de se obterem tais estimativas futuras, o uso de painel de especialistas é especialmente útil na validação e adaptação de dados internacionais e na gama de valores plausíveis para uma análise de sensibilidade 15 .

\section{Apresentação dos resultados}

Os resultados da AIO devem ser apresentados sob a forma de um relatório acompanhado dos arquivos das planilhas eletrônicas usados na análise principal e na análise de sensibilidade. O relatório deve ser redigido de forma objetiva e concisa, mencionando a origem das informações e enunciando pressupostos assumidos nas estimativas das variáveis do modelo de impacto orçamentário. Quando uma árvore de decisão ou modelo de Markov for empregado em substituição à planilha de impacto orçamentário, o arquivo eletrônico do método utilizado deverá ser fornecido. O relatório e seus anexos em formato eletrônico deverão ser claros e conter informações suficientes que permitam a um revisor recompor as diferentes etapas da análise.

\section{Validação e verificação da acurácia do modelo de impacto orçamentário}

A realização de testes da acurácia dos critérios adotados nas modelagens de impacto orçamentário usando dados reais oferecerá credibilidade às estimativas obtidas. Testes de validação são realizados aplicando-se dados históricos para verificar se o modelo desenvolvido previu adequadamente o comportamento dos valores estimados. Desse modo, o modelo poderá ser ajustado para melhor refletir o comportamento dos dados reais (calibragem do modelo). Embora a realização de testes de validação aumente a confiança nas estimativas obtidas, ela não é uma etapa obrigatória na execução de uma AIO.

\section{Comparação da proposta brasileira para estudos de impacto orçamentário com as diretrizes internacionais}

Nas duas últimas décadas, diversos países emitiram recomendações nacionais quanto ao desenvolvimento dos estudos de impacto orçamentário, destacando-se Canadá, Austrália, Polônia e Reino Unido. Em 2007, a sociedade internacional para pesquisa em desfechos farmacoeconômicos (ISPOR) emitiu recomendações referentes às particularidades metodológicas das AIO.

As principais particularidades dessas recomendações foram as seguintes:

- Todas se referem a impacto financeiro de fármacos, sem considerações quanto à avaliação de outras tecnologias da saúde.

- Todas indicam a realização de estudos de impacto orçamentário usando planilhas eletrônicas.

- Canadá e Austrália apresentam sistemas de saúde divididos em planos de medicamentos regionais ou provinciais, o que gera a necessidade de estudos de impacto orçamentário específicos para os planos de medicamentos.

- Canadá, Austrália e Polônia preveem a possibilidade de reembolso parcial pelos tratamentos utilizados, havendo pagamento complementar por parte dos usuários do sistema de saúde.

- No Reino Unido, o impacto financeiro de uma nova tecnologia da saúde é considerado somente do ponto de vista contábil, não fazendo parte do processo de avaliação de uma tecnologia em saúde, não sendo, portanto, considerado na decisão por incorporação ou não de novas opções terapêuticas.

A proposta brasileira assemelha-se às recomendações canadenses e australianas para a AIO de fármacos. À semelhança dessas, também foram elaboradas planilhas eletrônicas que funcionam como roteiro e como ferramenta para cálculo do impacto orçamentário. As recomendações brasileiras apresentam as seguintes diferenças principais em relação às recomendações internacionais:

- Fornecem orientações para a estimativa do impacto financeiro não somente de fármacos, mas também de outras tecnologias da saúde.

- É oferecida a possibilidade da realização de estudos de impacto orçamentário sob a perspectiva do SUS em suas diferentes instâncias, o que inclui gestores federal, estadual, municipal e saúde suplementar. 
- Não é prevista a possibilidade de custeio complementar pelos usuários do sistema de saúde, visto que, no SUS, todo o custo dos tratamentos é financiado pelo orçamento nacional para a saúde, com recursos captados por meio de impostos.

\section{Considerações finais}

A AIO deve ser considerada como parte integrante das avaliações de tecnologias em saúde no cenário brasileiro; recomenda-se que seja empregada sempre que uma nova tecnologia demonstrar comprovadamente sua segurança, eficácia, efetividade e, possivelmente, a relação custo-efetividade. A aplicabilidade do método no Brasil tornou-se mais favorável com a publicação da Lei $n^{o}$. 12.401/2011 16, que estabelece novas regras para a incorporação de tecnologias em saúde no âmbito do SUS.

Considerando que a AIO é um método mais recente e que ainda está em desenvolvimento e aperfeiçoamento por pesquisadores de todo o mundo, as atuais recomendações deverão ser revisadas para contemplar futuros avanços metodológicos.

\section{Resumo}

Análises de impacto orçamentário (AIO) fornecem previsões financeiras operacionais para a implementação de uma nova tecnologia em um sistema de saúde. Até o momento, não havia recomendações específicas para o desenvolvimento de AIO no Brasil. O presente trabalho apresenta uma revisão dos métodos usados nas AIO de tecnologias da saúde e propõe recomendações para seu uso nos âmbitos do SUS e do sistema de saúde suplementar. As recomendações principais são: analisar pela perspectiva do gestor; empregar horizonte temporal de um a cinco anos; comparar cenários de referência e alternativos; considerar taxa de incorporação da tecnologia; delimitar a população de interesse pela abordagem epidemiológica ou por demanda aferida; considerar restrições para acesso ou fatores indutores de demanda; considerar custos diretos incorridos e evitados; não ajustar para inflação ou descontos; integrar as informações em planilha eletrônica; calcular o impacto orçamentário diferencial entre cenários; sintetizar as informações em um relatório de impacto orçamentário.

Diretrizes para o Planejamento em Saúde; Financiamento em Saúde; Avaliação em Saúde 


\section{Colaboradores}

A. L. Ferreira-Da-Silva contribuiu com a revisão da literatura, redação das versões original e final, estudo detalhado e tradução das diretrizes internacionais de impacto orçamentário, desenvolvimento de planilha eletrônica para estimativas de impacto orçamentário e avaliação e incorporação das sugestões dos outros autores, como também de consultores. R. A. Ribeiro contribuiu com a revisão da literatura e redação do texto original, desenvolvimento de planilha eletrônica para estimativas de impacto orçamentário e revisão sobre modelagem farmacoeconômica (modelos de Markov). V. C. C. Santos participou da revisão das versões de trabalho, contribuiu com texto original sobre a avaliação de tecnologias em saúde no Brasil e com consultoria referente aos ajustes econômicos. F. T. S. Elias participou da revisão das versões de trabalho, apresentou contribuições originais ao texto final, sobretudo sobre métodos empregados internacionalmente para resolução de dilemas ético-técnicos, e participou da revisão dos aspectos legais - legislação na área de avaliação de tecnologias da saúde. A. L. P. d'Oliveira colaborou com a revisão das versões de trabalho e com ideias originais sobre métodos para impacto orçamentário. C. A. Polanczyk deu contribuições originais na versão final e participou do estudo detalhado das diretrizes internacionais de impacto orçamentário, do desenvolvimento de planilha eletrônica para estimativas de impacto orçamentário e da avaliação e incorporação das sugestões dos outros autores e de consultores.

\section{Referências}

1. Mauskopf JA, Sullivan SD, Annemans L, Caro J, Mullins CD, Nuijten M, et al. Principles of good practice for budget impact analysis: report of the ISPOR Task Force on good research practices - budget impact analysis. Value Health 2007; 10: 336-47.

2. Neumann PJ. Budget impact analyses get some respect. Value Health 2007; 10:324-5.

3. Marshall DA, Douglas PR, Drummond MF, Torrance GW, MacLeod S, Manti O, et al. Guidelines for conducting pharmaceutical budget impact analyses for submission to public drug plans in Canada. Pharmacoeconomics 2008; 26:477-95.

4. National Institute for Health and Clinical Excellence. Developing costing tools - methods guide. London: National Institute for Health and Clinical Excellence; 2008.

\section{Agradecimentos}

Agradecimentos à equipe do Instituto de Avaliação de Tecnologia em Saúde (IATS), em especial a Juliana Juk.
5. Pharmaceutical Benefits Advisory Committee. Guidelines for preparing submissions to the Pharmaceutical Benefits Advisory Committee. Canberra: Department of Health and Ageing, Australian Government; 2006.

6. Orlewska E, Mierzejewski P. Proposal of Polish guidelines for conducting financial analysis and their comparison to existing guidance on budget impact in other countries. Value Health 2004; 7: 1-10.

7. Ministério da Saúde. Diretrizes metodológicas: estudos de avaliação econômica de tecnologias da saúde. Brasília: Ministério da Saúde; 2009.

8. Nuijten MJC, Ruten F. Combining a budgetary impact analysis and a cost-effectiveness analysis using decision-analytic modelling techniques. Pharmacoeconomics 2002; 20:855-67. 
9. Departamento de Ciência e Tecnologia, Ministério da Saúde. Informe técnico institucional. Avaliação de tecnologias em saúde: institucionalização das ações no Ministério da Saúde. Rev Saúde Pública 2006; 40:743-7.

10. HTA Core Models Handbook. https://fio.stakes. fi/htacore/handbook.html (acessado em 19/ Jun/2011).

11. Orlewska E, Gulacsi L. Budget-impact analyses: a critical review of published studies. Pharmacoeconomics 2009; 27:807-27.

12. Sonnenberg FA, Beck JR. Markov models in medical decision making: a practical guide. Med Decis Making 1993; 13:322-38.

13. Vianna CMM, Caetano R, Ugá MA. Diretrizes metodológicas para estudos de avaliação econômica de tecnologias em saúde. Brasília: Ministério da Saúde; 2009.
14. Drummond FD, Sculpher MJ, Torrance GW, O'Brien BJ, Stoddart GL. Methods for the economic evaluation of health care programmes. $3^{\text {rd }}$ Ed. New York: Oxford University Press; 2005.

15. Nuijten MJC, Mittendorf T, Persson U. Practical issues in handling data input and uncertainty in a budget impact analysis. Eur J Health Econ 2010; 12:231-41.

16. Brasil. Lei n ${ }^{\circ}$. 12.401, de 28 de abril de 2011. Diário Oficial da União 2011; 29 abr.

Recebido em 27/Jul/2011

Versão final reapresentada em 28/Fev/2012

Aprovado em 02/Abr/2012 